\title{
A systematic scoping review of the molecular mechanisms underpinning phytosterol and phytostanol mediated epigenetic changes
}

\author{
E. Jefrei ${ }^{1}$, M. Xu ${ }^{1}$, J.B. Moore ${ }^{1}$ and J.L. Thorne ${ }^{1}$ \\ ${ }^{1}$ School of Food Science and Nutrition, University of Leeds, Leeds, UK
}

Nutrition is perhaps one of the most fundamental and well understood modifiers of epigenetic inheritance. Epigenetics is meiotic or mitotic inherited change to gene expression, induced by a transient founder event, that does not involve alterations to the genetic sequence. Molecular mechanisms conferring epigenetic inheritance include DNA methylation via DNA methyltransferase enzymes (DNMT), post-translational modifications of histones such as histone deacetylases (HDAC), and micro-RNAs. Phytosterols/phytostanols (PSS) are nutrients found in vegetable oils, nuts, seeds, and also added to a range of commercial dairy products. PSS reduce circulating LDL-cholesterol and have been linked with lower risk of several non-communicable diseases ${ }^{(1,2)}$. Individuals who consume plant sterols typically do so as part of a chronic long-term dietary exposure pattern, ideal for direct and indirect whole-body epigenetic changes to manifest. Our aims were to evaluate the current state of understanding regarding how PSS modify epigenetic molecular mechanisms and determine the range of evidence whether epigenetic modifications are involved in mediating the protective effects of PSS against non-communicable diseases.

A systematic search was performed in PubMed, Scopus, and Web of Science using phytosterol and epigenetics related keywords and adhering to PROSPERO criteria. After removal of duplicates, 226 abstracts were screened for eligibility by two independent researchers. Discordance was resolved by a third researcher. Eight records were eligible for information extraction.

PSS regulate a range of micro-RNAs (four studies) including nine that were regulated in two or more studies, inhibit HDAC activity (three studies), and impair DNMT expression and activity (two studies). Two studies (in rat neurons and human endothelial cells) that screened miRNA, each reported more than 100 miRNAs had $>2$-fold differential regulation following PSS exposure. PSS have also been found to be potent chemical inhibitors of class I HDACs ${ }^{(3)}$. Interestingly this had previously been independently predicted by a computational modelling group ${ }^{(4)}$. Finally, both expression and activity of DNMT enzymes was inhibited by PSS in prostate and breast cancer cell lines.

In conclusion, we found that although PSS are likely to exert mitotic epigenetic inheritance in mammalian cells via the typical epigenetic molecular mechanisms of action, evidence of meiotic/transgenerational inheritance was not found. Our results indicated that PSS inhibit histone deacetylation enzymes in multiple cell and tissue types, suggesting they directly bind and allosterically impair HDAC activity. PSS are also likely to activate gene expression, at least in cancer cell lines, via their ability to inhibit DNMT enzymes and alter expression of miRNAs. Although epigenetic changes are typically cell type specific, this scoping review has revealed that too few studies have been conducted in this field to identify similarities or differences between cell types.

\section{References}

1. Cioccoloni G, et al. (2020) Crit Rev Food Sci Nutr 1-21.

2. Plat J, et al. (2019) Prog Lipid Res 74, 87-102.

3. Ishola AA \& Adewole KE (2019) Mol Biol Rep 46, 2307-2325.

4. Shao Y, et al. (2016) Molecules 21. 